\title{
A serious game for training balance control over different types of soil
}

\author{
Bob-Antoine J. Menelas, Martin J. D. Otis
}

\begin{abstract}
It is known that the type of the soil can affect balance. Here we report a serious game designed for training users at maintaining balance over five types of soil (broken stone, stone dust, sand, concrete and wood). By using an augmented shoe and proposed navigation metaphor, in this game, the user is invited to browse a maze with standing balance over the physical grounds. During the exploration, exercises targeting assessment balance control are suggested. To insure the effectiveness of this training program, four exercises based on the Berg Balance Scale and the Tinetti Balance Assessment Tool are incorporated in the game.
\end{abstract}

\section{Introduction}

Falls represent a major factor in the frail elderly. In developed countries, we observed that nearly half of falls among elderly caused minor injury while 5 to $25 \%$ sustained serious damages such as fracture of the proximal femur [9]. Beyond the physical injuries, in many cases, falls leave a psychological impact due to the fear of falling. As a consequence, even without an injury, a fall can cause a loss in confidence and a reduction of mobility. This can lead to a decline in health and function and contribute to future falls with more serious outcomes [5]. These observations have promoted the development of multiple programs dedicated to prevention of accidental falls. Given the multifactorial aspect of falls, these programs have to target several factors that can constitute a certain risk of fall [3]. In this way, several programs have coupled the practice of physical exercises to analysis of balance and

B.-A. J. Menelas

University of Quebec at Chicoutimi (UQAC), Department of Mathematics and Computer Science, Quebec, Canada, e-mail: bamenela@uqac.ca

M. J.-D. Otis

University of Quebec at Chicoutimi (UQAC), Department of Applied Sciences, REPARTI Center, Quebec, Canada, e-mail: Martin_Otis@uqac.ca 
gait. Others have been focused on control of vision, hearing and blood pressure. Our research project aims at taking all these factors and even several others into account. Here, we consider training balance control via exercise programs.

Balance control via exercises has been an active research topic in the last decade, multiples approaches have been proposed. However, to the best of our knowledge, no research has yet integrated the type of soil in training programs targeting improvement of balance control. Given that the type of soil, that a person walks on, may affect his balance [17], our work aims at helping users at maintaining their balance over several types of soil by using a serious game.

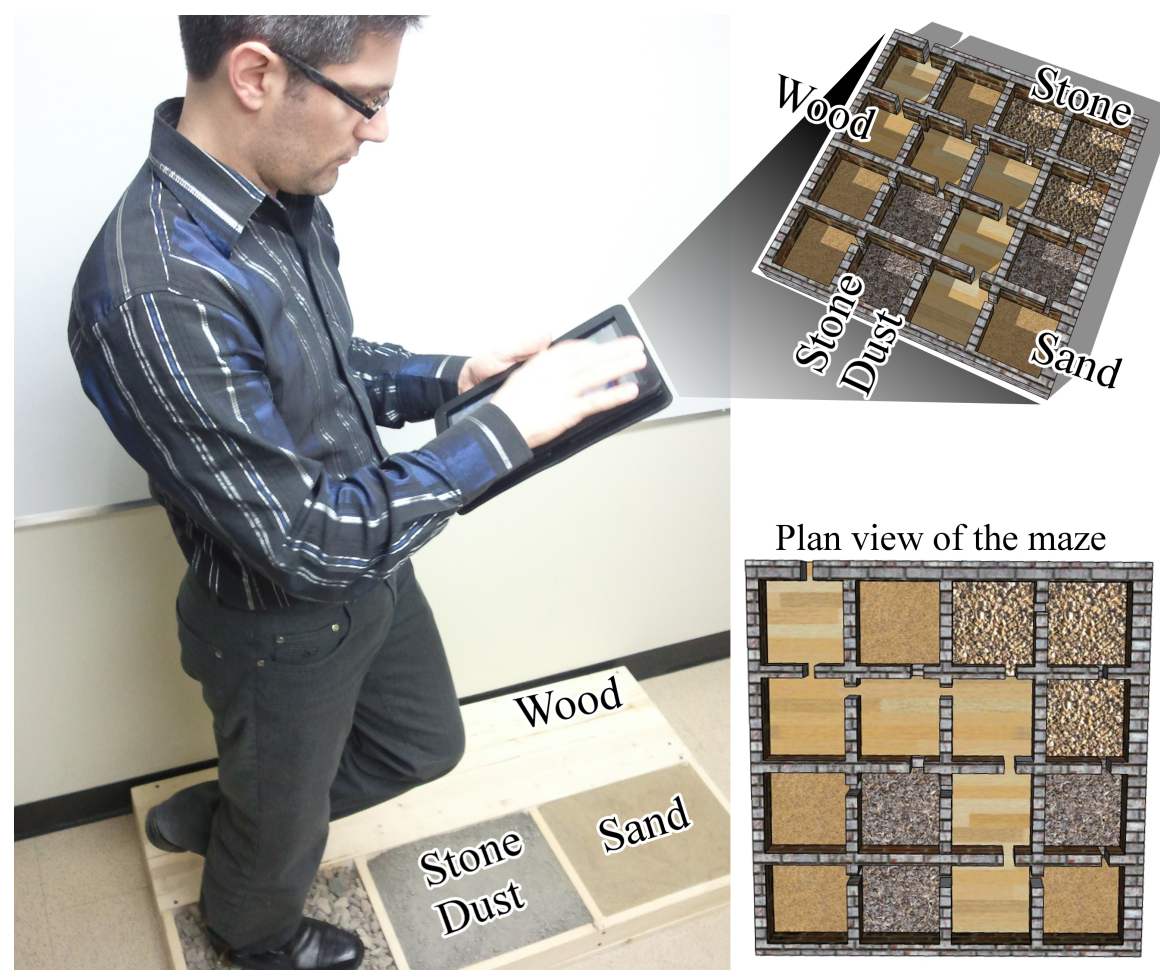

Fig. 1 A user is playing the game while standing on one leg.

By playing this game, we want to bring the user to experiment balance control over several types of soil: broken stone, stone dust, sand, concrete and wood. To insure the ecological validity of the system, it combines elements of the real world, to an interactive virtual environment. The game is designed in order to be usable for the realization of home exercises. In the proposed game, the user is invited to browse a virtual maze having five types of ground (see Fig. 1). During the exploration, fitted with an augmented shoe, the user has to face several distracting stimuli designed in order to bring him at realizing movements similar to those that occur at the beginning of a spontaneous loss of balance. Delivered stimuli are based on 
exercises targeting balance assessment (Berg Balance Scale and the Tinetti Balance Assessment Tool). During this process, various measures relative to the user's posture and its heart rate are measured in order to check how the situation affects the user.

The paper is organized as follows: related work are presented at section II, the developed serious game is detailed in section III and the conclusion in section IV.

\section{Related work}

Various studies have proved that balance control plays an important role in fall prevention. As a result, it has been incorporated into multiple programs aiming at fall prevention. Previous researches show that practice of exercises can reduce the risk of fractures. By helping to maintain bone mass, it allows to improve notably the stability posture. Madureira et al. have shown that balance training program is effective in reducing the risk of falling in elderly [16]. Traditionally exercise programs were delivered in a class situation or individually by professional physical therapist $[18,19]$.

To alleviate several constraints regarding such an approach (cost of transportation, schedule management, etc.) new methods that use some technological devices have emerged. Exploited approaches range from pure visual system (like the Nintendo Wii-Fit) to vibrotactile feedback embedded in a cell phone $[8,10,14]$. In [8], a 6-week home-based balance training program using Nintendo Wii Fit and balance board is presented. In the same way, Grosjean et al. have combined the Nintendo Wii Fit platform to traditional physical therapy interventions [10]. Also, Lee et al. have described a cell phone based vibrotactile feedback system dedicated to balance rehabilitation training [14]. Realized experiment shows that real-time feedback provided via a cell phone can be used to reduce body sway.

Looking at the literature, we observed that physical characteristics of the soil have not been considered for training of balance control. For person with gait disorder or losing autonomy, it appears that walking on different type of soil could be a challenge and may represent a risk of falling. Moreover, it has been demonstrated that the type of soil may affect the gait [17]. As result, it seems quite important to help users at maintaining their balance over different types of soil. Considering the promising results of cited studies, we have developed an interactive game for balance training over different types of soil.

The proposed game combines elements of the real world, to an interactive virtual maze. By exposing the user to various destabilizing events (perturbations) the game aims at helping users strengthen their lower limbs while learning facing perturbations that can occur in daily activities. Moreover, by using an interactive shoe, the dynamic of the users and his ability to maintaining postural stability after a perturbation are logged in real time. In the following, we describe the game. 


\section{Proposed Game}

With this game, we want to design an environment that can help users at training their balance over several types of soil. Given that successful previous balance training programs have usually run over a long period [16], we designed the game in a way so that a user can experiment it over a long period. Among several other requirements, it has been designed to be fun, interactive, safe, ease to use and usable at home.

In this game, the player is invited to browse a virtual maze as fast as possible while standing balanced on a specific type of soil. To do so, fitted with an augmented shoe, to move in the maze and to perform exercices, the player remains physically at the same position and uses the metaphor presented in subsection 3.1.1.

\subsection{System overview}

The game presented in this document requires a physical setup and an instrumented shoe. This section briefly summarizes these two components.

\subsubsection{Interactive shoe}

Recently, we have developed an intelligent system that aims at prevent accidental falls related to conditions of the physical environment of the person (slippery ground, steep slope, etc.), or abnormalities of its gait. This system is centered on an augmented shoe (right part of Fig. 2). This device counts, on one side, a set of sensors that serves for characterizing the dynamics of walking, the posture of the user and the physical properties of the environment. For example, they measure the velocity and acceleration of the foot, bending of the sole and forces applied at five points under the foot (as seen in Fig. 3). All these sensors are exploited to compute the risk level associated to physical characteristics of the environment. On the other hand, this device brings together several actuators aiming to transmit vibrotactile signals to the user. These signals intend to attract the attention of the user towards situations deemed dangerous by the control system running on a Android device (tablet or smartphone). For more details regarding the interactive shoe, one can refer to models of instrumented shoes like [1], [7] and [20]. As part of this game, for each soil type, we used these sensors to measure the variation of several parameters (acceleration, sole bending, applied force by the user, etc.) when maintaining or recovering balance.

Another sensor used in the system is a portable wireless device that helps to measure the electric activity of the heart. This heart rate monitor is designed for cardiovascular training - running, jogging, or any kind of strenuous exercise. Its main component is a heart rate monitor strapped over the chest with bluetooth capabilities. This device reads and transmits the heart rate to the Android device. 

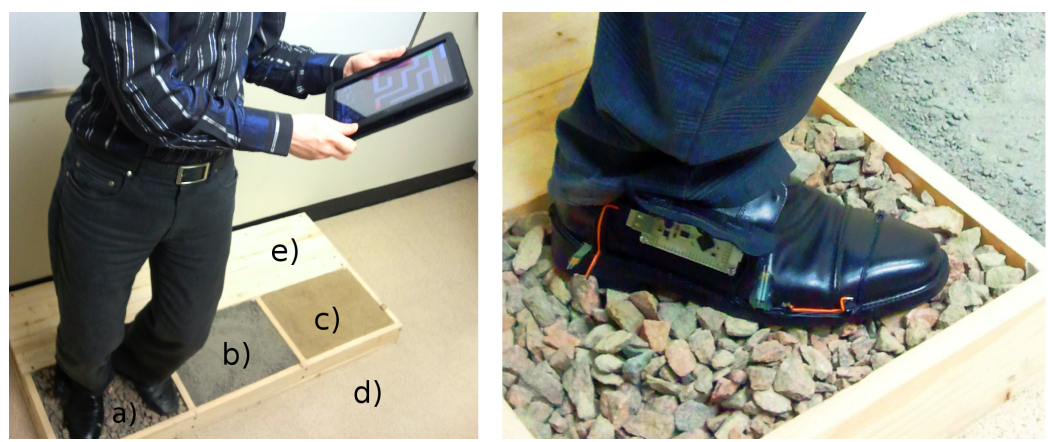

Fig. 2 Setup used for training of balance control (left side). Interactive shoe (right side).

\subsubsection{Physical materials}

Our work intends to fulfill the gap observed in the literature about balance training over different types of soil. To ensure the ecological validate of this work, even though we propose a serious game, we wanted to use physical soils. Moreover, in order to cover a wide range of soil, three granular (deformable) and two nondeformable materials were used as type of ground. The three granular materials are broken stone, sand and dust stone whereas concrete and wood were the non deformable. These materials have been selected because of their rheological characteristics and their relatively high rate of use in living environments. In the context of the game, they spread out in a tray as shown in Fig. 2.

\subsection{Metaphor used for the displacement in the game}

In the proposed game, the user has to browse, as fast as possible, a virtual maze while standing balanced. Since the goal behind the game is to improve balance control over different types of soil; it seems appropriate to exploit body interactions for displacements in the maze. Moreover, it has been showed that such interactions may enhance the engagement of users in the games [2].

For this, interfaces such a Microsoft Kinect ${ }^{T M}$ could be considered. Indeed, a recent study used the Microsoft Kinect ${ }^{T M}$ to assess kinematic strategies of postural control [4]. However, such a system does not really fit our need since it can not be used to evaluate all aspect regarding the balance of the user of different types of soil. We therefore have prefer our device; it includes several sensors that can be used for evaluate the balance of the user.

The navigation metaphor used is based on the repartition of the forces applied to the sole of the shoe. To navigate the user just has to swing (see Fig. 4). As shown in Fig. 3, two types of sensor measure the forces reparation in sole: the FSR force sensors and the bending sole. Therefore two components let to compute the dis- 


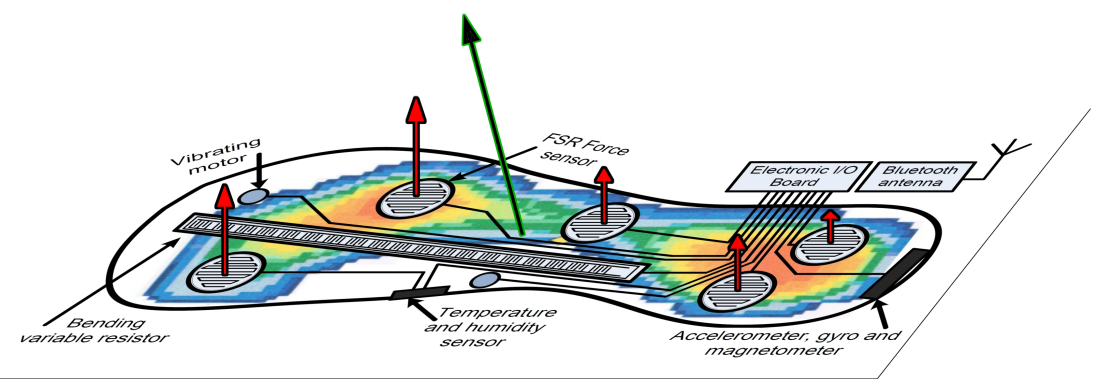

Fig. 3 Instrumented sole exploited for navigation in the game. Arrows in red are the force measurement at each sensor. The sum of these arrows is represented by the black arrow.

placement of the avatar in the scene. The first uses the direction given by the vector forces computed by the (FSR) force sensors. The second component measures the angle of the sole bending to produce a proportional movement in linear direction (forward) (see Fig. 5). These components are determined as follow:

- The total force and moment applied on the sole by the user generate a wrench $\mathbf{h}_{a} \in \mathfrak{R}_{6 \times 1}$ which is computed by equation (1). The position $A$ is the reference frame in the game, $B$ is the reference frame of the shoe, ${ }^{A} Q_{B} \in \Re_{3 \times 3}$ is the rotation matrix between reference frame $A$ and its counterpart $B$. Also, $m$ is the number of sensors inserted in the sole at the position $\mathbf{q}_{i} . B$ is located at the center of pressure applied on the sole in a upright posture of the user. The vector $\mathbf{h}_{r}$ is a projection of the vector $\mathbf{h}_{a}$ on the plane described by the virtual ground which gives the direction of avatar's movement as seen in Fig. 6. The amplitude of the projected vector gives a proportional movement in the maze. In the current version, a Hooke constant $\mathbf{k}$ is used to compute a variation of displacement as described by the equation (2). The current position of the avatar is $\mathbf{s}_{a}^{n}$, at the discrete time $n$.

- The sole bending is evaluated with a bending variable resistor which gives only one angle $\theta$ as shown in Fig. 5. This angle is thus used to move forward. Since the bending is proportional to an angle $\theta$, we compute a displacment in the maze $s_{y}^{n}$ with the equation (4) using a proportional constant $k_{\theta}$ which reflects the sole curvature into a linear movement.

$$
\begin{aligned}
& \mathbf{h}_{a}=\left[\begin{array}{c}
\sum_{i=0}^{m-1}{ }^{\mathbf{A}} \mathbf{Q}_{\mathbf{B}} \mathbf{f}_{a_{i}} \\
\sum_{i=0}^{m-1}\left(\mathbf{q}_{i} \times\left({ }^{\mathbf{A}} \mathbf{Q}_{\mathbf{B}} f_{a_{i}}\right)\right)
\end{array}\right] \\
& \mathbf{s}_{a}^{n}=\mathbf{s}_{a}^{n-1}+\mathbf{h}_{r} / \mathbf{k} \text {, with }
\end{aligned}
$$

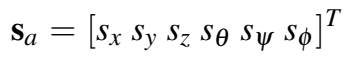



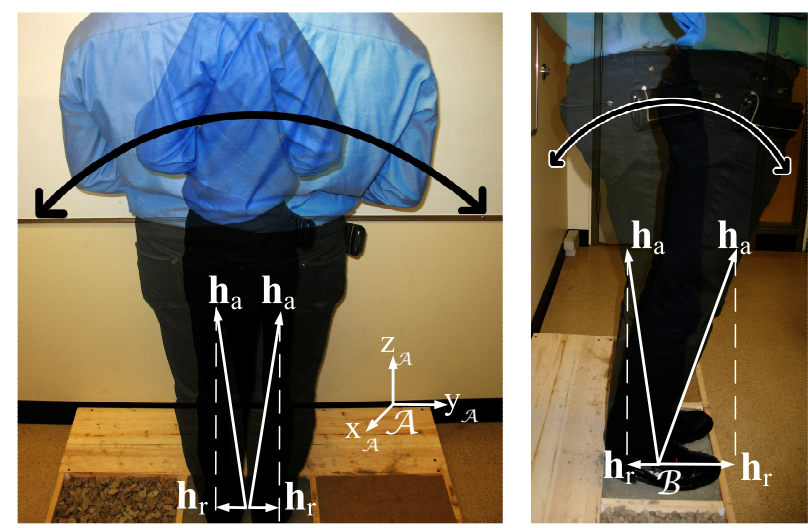

Fig. 4 Displacement with the navigation metaphor. Any swing of the user does generate a displacement in the maze.

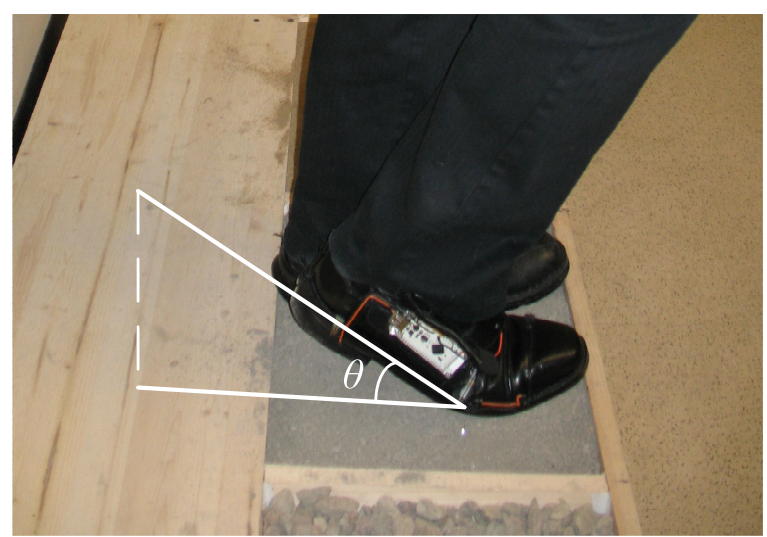

Fig. 5 Representation of the direction coming from the bending of the sole.

$$
s_{y}^{n}=s_{y}^{n-1}+k_{\theta} \theta
$$




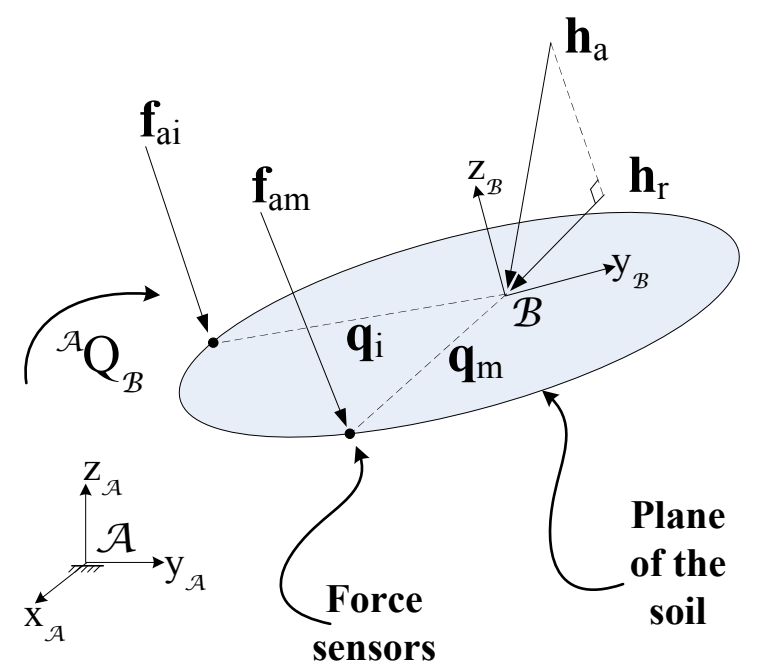

Fig. 6 Movement computation in the game used as navigation metaphor

\subsection{Balance control: assement and training}

The goal of the game is to train people on maintaining balance on different types of soil. Because of that, assessment of the ability of the person to maintain balance plays an important role in the game. A special attention was paid to selection of exercises that can serve this goal.

Literature on assessment of the ability to maintain balance counts two main categories: 1) exercises or tasks based evaluation and 2) the patient's health. Our game is centered on exercises based evaluation.

Berg Balance Scale (BBS) and Morse Fall Scale (MFS) are two main tools used for fall risk prediction [22]. These tests are very different since the BBS analyzes some tasks (exercises) whereas the MFS approach analyzes the health and the gait of the patient. On the other hand, it was demonstrated that the Heindrich II Fall Risk Model (HFRM) is potentially useful in identifying patients at high risk for falls; some studies suggest that it can be more relevant than both the BBS and the MFS [13]. However, the HFRM approach concerns the analysis of the patient' health and do not cover exercises [11]. The MFS and the Functional Reach test was found to be time consuming and often inconvenient and were not better at prediction than the clinical judgments made by the primary nurses [6]. Finally, the Tinetti Balance Assessment Tool (TBAT) (described in [21]) was also compare with Functional Reach. TBAT seems to be the most suitable performance measure for evaluating balance in community-dwelling older people [15]. A comprehensive review of methods to assess the risk of a fall is presented in [12]. 
As seen through that brief analysis, Berg Balance Scale and the Tinetti assessment tool appear at most appropriate tests for exercises based evaluation. We have therefore integrated exercises proposed in these tests in our game. Proposed exercises are associated with some parts of the maze. During the browsing of the maze, while entering a section associated to an exercise, all motions (used to move the avatar) are disable for the period of the exercise, a video presenting the exercise to realize is shown to the user. In the proposed game, selected exercises are:

- Standing unsupported with feet together;

- Turning in place;

- Placing alternate foot on step;

- Standing on one leg;

With the navigation metaphors (presented in section 3.2) and the sensors located in the sole of the interactive shoe (presented in section 3.1.1), it is possible to differentiate the four selected exercices and compute a score according to the success of the exercises.

\subsection{Different levels of difficulty}

The complexity of the game could be described by two components: the maze it-self (number of paths, size of the maze and number of dead end) and proposed exercises. At the current version of the game, we do have only rectangular mazes: proposed route are only vertical or horizontal. Each little rectangle described by the route defines a particular zone and is therefore associated with a particular type of soil (sand, stone, dust stone, wood and concrete). As a result, in the current version of the game, the complexity is mainly determined by the complexity of the exercise to achieve on given type of soil. In general, MFS, TBAT and HFRM use three levels of risk (low, moderate and high). Since we use an additional level of risk in the game (type of soil), four levels of risk (low, moderate, high and very high) are implemented in the game.

At the current stage, the game counts three levels of difficulty. At the easiest level, user is invited to stand on both feet, there is no time constraint for the completion of the task and proposed mazes are the easiest. At the intermediate level, the task has to be completed in a certain amount of time while the mazes are a little more complicated. Although sometime the user has to stand on one foot, most of time he has to stand on both feet. At the hardest level, the time allocated for the completion of the task is reduced and at the meantime the mazes become highly interactive while presenting various obstacles to the user. At this level, when gifted with some bonuses, the user has the possibility to overcome certain constraints of the maze. For example, he may jump over a wall by placing alternate foot on step (the apparatus used for holding the soils as described in section 3.1.2). At this step, all the time the user has to stand on one foot. As a result, the use of both feet is seen as a mistake and generates a reduction of the score. 
These three levels are designed in order to provide a progressive level of difficulty that will help the user familiarizing themselves to situation that can lead to accidental falls.

\subsection{Score}

To maintain the engagement of the player in the game, we consider all the factors described previously to evaluate a score. The score displayed in real-time is computed according to the execution of the exercise, the difficulty level and the time.

As suggested in the Berg Balance Scale, each exercise is rated on a scale of 0 to 4. The distribution of the forces measured in the sole determines the value accorded for the exercise. In fact, the computed value is inversely proportional to the standard deviation $\sigma$ of the measured force $\left|\mathbf{h}_{a}\right|$ over the period of the exercise. Hence, when doing an exercise, the user has to stay in a fixed position in order to maximize his score: the more he swings the lower his score for the exercise will be. Each soil type has a difficulty level labelled $D$. The difficulty level associated for soil types are: concrete (or wood): 1 , stone dust: 2 , stone: 3 and sand: 4 . To compute the final score, for each exercise, this difficulty level is multiplied by the score of the exercise. When the time of execution is considered in the game, the final value is inversely proportional to the time $t$ with a scale factor $\alpha$, as described by equation (5):

$$
\text { Score }=\frac{D}{\sigma(\alpha t)}
$$

This score aims at evaluating the level at which the user is able to maintain its balance on different types of soil.

\subsection{Safety issue}

One of the main advantages of using a serious game for balance training resides in the fact that the game can be experimented at home. However, this raises a number of problems related to player's safety. For this, several design choices were made in order to guaranty the safety of the player.

Although the game was designed to train and practice balance control, players should be aware that some of the proposed exercises may represent a risk. For example, previously we mentioned that using deformable soils increase the risk of falling. More particularly, standing on one foot over the sand soil could be challenging for people with balance disorder. Furthermore, the current platform requires a location in a room free from obstructions that may hinder user's movements. Beyond these aspects, in order to prevent potential injuries, at the end of the analysis performed on the data logged from the ECG and those concerning the posture of the player, if a serious trouble is detected, the game is locked and invites the player to 
contact the costumer service because the game seems not to be appropriate to his profile.

\section{Conclusion and Future Work}

This paper described a serious game for training balance control over five types of soil (broken stone, stone dust, sand, concrete and wood). To provide an efficient training program, proposed exercises, used to assess the balance of the user, are selected from standard evaluation test. With the current version of the game, we are able to record various parameters that will inform us about the dynamic of balance control over different types of soil. Doing so, the game will let us identifying parameters which could lead the user to a loss of balance. This information will be useful for the design of a new generation of enactive shoe dedicated to preventing fall. Then, the shoe will use these parameters in daily activities (real situation) for warning users whenever a potentially dangerous situation occurs. The warning signal can be generated via the actuators, located inside the shoe, which are able to synthesis different types of signal such as ecological icons.

\section{References}

1. Bamberg, S., Benbasat, A., Scarborough, D., Krebs, D., Paradiso, J.: Gait analysis using a shoe-integrated wireless sensor system. IEEE Transactions on Information Technology in Biomedicine 12(4), 413-423 (2008)

2. Burke, J., McNeill, M., Charles, D., Morrow, P., Crosbie, J., McDonough, S.: Optimising engagement for stroke rehabilitation using serious games. Visual Computer pp. 1-15 (2009)

3. Chang, J.T., Morton, S.C., Rubenstein, L.Z., Mojica, W.A., Maglione, M., Suttorp, M.J., Roth, E.A., Shekelle, P.G.: Interventions for the prevention of falls in older adults: systematic review and meta-analysis of randomised clinical trials. BMJ 328(7441), 680+ (2004). DOI 10.1136/bmj.328.7441.680. URL http://dx.doi.org/10.1136/bmj.328.7441.680

4. Clark, R., Pua, Y.H., Fortin, K., Ritchie, C., Webster, K., Denehy, L., Bryant, A.: Validity of the microsoft kinect for assessment of postural control. Gait and Posture (2012)

5. Cumming, R., Salkeld, G., Thomas, M., Szonyi, G.: Prospective study of the impact of fear of falling on activities of daily living, sf-36 scores, and nursing home admission. Journals of Gerontology - Series A Biological Sciences and Medical Sciences 55(5), M299-M305 (2000)

6. Eagle, D., Salama, S., Whitman, D., Evans, L., Ho, E., Olde, J.: Comparison of three instruments in predicting accidental falls in selected inpatients in a general teaching hospital. Journal of gerontological nursing 25(7), 40-45 (1999)

7. Edgar, S., Swyka, T., Fulk, G., Sazonov, E.: Wearable shoe-based device for rehabilitation of stroke patients. In: International Conference of the IEEE Engineering in Medicine and Biology Society, pp. 3772-3775 (2010)

8. Esculier, J.F., Vaudrin, J., Beriault, P., Gagnon, K., Tremblay, L.: Home-based balance training programme using wii fit with balance board for parkinson's disease: A pilot study. Journal of Rehabilitation Medicine 44(2), 144-150 (2012)

9. Ganz, D., Bao, Y., Shekelle, P., Rubenstein, L.: Will my patient fall? Journal of the American Medical Association 297(1), 77-86 (2007) 
10. Grosjean, A., Fabbri, E., Feldheim, E., Snoeck, T., Amand, M., Keuterickx, C., Balestra, C.: On the use of the wii fit in reducing falling risk factors and improving balance for the elderly. Kinesitherapie 10(107), 41-45 (2010)

11. Hendrich, A., Nyhuis, A., Kippenbrock, T., Soja, M.: Hospital falls: development of a predictive model for clinical practice. Applied Nursing Research 8(3), 129-139 (1995)

12. Howe, T., Rochester, L., Neil, F., Skelton, D., Ballinger, C.: Exercise for improving balance in older people. Cochrane database of systematic reviews (Online) CD004963(11), 1-152 (2011)

13. Kim, E., Mordiffi, S., Bee, W., Devi, K., Evans, D.: Evaluation of three fall-risk assessment tools in an acute care setting. Journal of Advanced Nursing 60(4), 427-435 (2007)

14. Lee, B.C., Kim, J., Chen, S., Sienko, K.: Cell phone based balance trainer. Journal of NeuroEngineering and Rehabilitation p. 10 (2012)

15. Lin, M.R., Hwang, H.F., Hu, M.H., Wu, H.D., Wang, Y.W., Huang, F.C.: Psychometric comparisons of the timed up and go, one-leg stand, functional reach, and tinetti balance measures in community-dwelling older people. Journal of the American Geriatrics Society 52(8), 13431348 (2004)

16. Madureira, M.M., Takayama, L., Gallinaro, A.L., Caparbo, V.F., Costa, R.A., Pereira, R.M.R.: Balance training program is highly effective in improving functional status and reducing the risk of falls in elderly women with osteoporosis: a randomized controlled trial. Osteoporosis International 18(4), 419-425 (2007)

17. Marigold, D., Patla, A.: Adapting locomotion to different surface compliances: Neuromuscular responses and changes in movement dynamics. Journal of Neurophysiology 94(3), 17331750 (2005)

18. Means, K.M., Rodell, D.E., O'Sullivan, P.S.: Balance, mobility, and falls among communitydwelling elderly persons: effects of a rehabilitation exercise program. American Journal of Physical Medicine and Rehabilitation 84(4), 238-250 (2005)

19. Nitz, J.C., Choy, N.L.: The efficacy of a specific balance-strategy training programme for preventing falls among older people: a pilot randomised controlled trial. Age Ageing 33(1), 52-58 (2004)

20. Noshadi, H., Ahmadian, S., Hagopian, H., Woodbridge, J., Dabiri, F., Amini, N., Sarrafzadeh, M., Terrafranca, N.: Hermes: Mobile balance and instability assessment system. In: Proceedings of the 3rd International Conference on Bio-inpsired Systems and Signal Processing, pp. 264-270 (2010)

21. Tinetti, M.: Performance-orientated assessment of mobility problems in elderly patients. Journal of the American Geriatrics Society 34(2), 119-126 (1986)

22. Zhou, J., Fan, J.: Analysis of the effectiveness of morse fall scale and berg balance scale applied in the fall risk prediction for senile patients. Chinese Journal of Rehabilitation Medicine 27(2), 130-133 (2012) 